\title{
AVALIAÇÃO DA QUALIDADE DAS PRESCRIÇÕES MEDICAMENTOSAS DISPENSADAS EM UMA UNIDADE DE SAÚDE DA FAMÍLIA NO MUNICÍPIO DE JOÃO PESSOA, PARAÍBA
}

Evaluation of the quality of medicinal prescriptions dispensed in a family health unit in the municipality of João Pessoa, Paraíba

Evaluación de la calidad de prescripciones de medicamentos rechazados en una unidad de salud de la familia en la ciudad João Pessoa Condado, Paraíba

Vanessa Domingos Morais ${ }^{1}$, Larissa Comarella², Jairo Domingos Morais ${ }^{3 *}$

1 Secretaria Municipal de Saúde de João Pessoa, Paraíba

2 Universidade Federal da Paraíba (UFPB)

${ }^{3}$ Universidade Federal do Paraná (UFPR)

*E-mail: jairodmfisio@hotmail.com

\section{RESUMO}

Introdução: No Sistema Único de Saúde (SUS), o acesso aos medicamentos se dá através da prescrição, que desempenha papel ímpar na prevenção de erros de medicação, já que é a partir dela que todo o fluxo de tratamento medicamentoso chega ao usuário.

Objetivo/Métodos: Dessa forma, o presente estudo do tipo transversal, retrospectivo descritivo, com abordagem quantitativa teve como objetivo a avaliação do cumprimento da legislação quanto à prescrição de medicamentos dispensadas pela farmácia da Unidade de Saúde da Família (USF) Nova Conquista.

Resultados: Foram analisadas 300 prescrições, com média de 1,49\% de medicamentos por prescrição. Os resultados apontaram a não totalidade dos critérios de avaliação proposto neste estudo dentre eles: presença de abreviaturas (98,05\%) e rasuras (7\%); ausência de informações como: endereço (98\%), forma farmacêutica (91,12\%), frequência e duração do tratamento (76,98\%) concentração do fármaco $(29,68)$, dose $(18,59 \%)$, posologia $(5,13 \%)$; unidade de medida ausente ou inadequada (1,80\%). As classes farmacológicas prevalentes foram: anti-inflamatórios não- esteroidais (28,04\%), anti-hipertensivo (14,15\%), antialérgicos $(11,51 \%)$ e antibióticos (8,73\%).

Conclusão: Todas as prescrições avaliadas neste estudo apresentaram alguma inconformidade ou ausência de informações em relação ao que estabelece a legislação, concluindo-se que alguns hábitos de prescrição devem ser modificados, buscando o melhoramento da qualidade da prescrição e do fortalecimento da assistência prestada no SUS.

Palavras-chave: Prescrições. Uso de medicamentos. Saúde pública.

\section{ABSTRACT}

Introduction: in the Unified Health System (SUS), access to medicines occurs through prescription, which plays a unique role in the prevention of medication errors, since it is from there that the entire flow of drug treatment reaches the user.

Objective/Methods: Thus, the present cross-sectional retrospective descriptive study, with a quantitative approach, aimed to evaluate compliance with the legislation regarding the prescription of medicines dispensed by USF Nova Conquista's pharmacy. Results: A total of 300 prescriptions were analyzed, with an average of $1.49 \%$ of medicines per prescription. The results pointed out not all of the evaluation criteria proposed in this study, among them: abbreviations (98.05\%) and erasures (7\%); concentration of the drug (29.68), dose (18.59\%), dosage (5,13\%), frequency and duration of treatment (76.98\%); unit of 
measurement was absent or inadequate (1.80\%). The prevalent pharmacological classes were: non-steroidal anti-inflammatory drugs (28.04\%), antihypertensive (14.15\%), antiallergic drugs (11.51\%) and antibiotics (8.73\%).

Conclusion: All prescriptions evaluated in this study presented some nonconformity or lack of information in relation to what the legislation establishes, and it is concluded that some prescribing habits should be modified, seeking to improve the quality of prescription and the strengthening of the assistance provided in the SUS .

Keywords: Prescriptions. Use of medications. Public health.

\section{RESUMEN}

Introducción: En el Sistema Único de Salud (SUS), el acceso a los medicamentos es a través de la prescripción, que juega un papel único en la prevención de errores de medicación, ya que es a partir de ella que todo el flujo de tratamiento de medicamentos llega al usuario.

Objetivo/Métodos: Por lo tanto, este estúdio, de tipo transversal, retrospectivo, descriptivo, de enfoque cuantitativo, tuvo el objetivo de evaluar el cumplimiento de la legislación sobre la prescripción demedicamentos rechazados por la farmacia de la Unidad de Salud de la Familia (USF) en Nova Conquista.

Resultado: Se analizaron 300 prescripciones, con un promedio de 1,49\% de medicamentos por prescripción. Los resultados mostraron la insuficiencia de los criterios de evaluación propuestos en este estudio. Entre ellos: la presencia de abreviaturas $(98,05 \%)$ y borrados (7\%); ausencia de información como: domicilio (98\%), forma farmacéutica (91,12\%), frecuencia y la duración del tratamiento (76,98\%), concentración del fármaco $(29,68)$, dosis $(18,59 \%)$, posología $(5,13 \%)$; unidad de medida ausente o inadecuada (1,80\%). Las clases farmacológicas prevalentes fueron: fármacos antiinflamatorios no esteroideos (28,04\%) antihipertensivo (14,15\%), antialérgicos $(11,51 \%)$ y antibióticos $(8,73 \%)$.

Conclusión: Todas las prescripciones evaluadas en este estudio mostraron alguna inconformidad o ausencia de información en relación a lo que dicta la ley, concluyendo que algunos hábitos de prescripción deben ser modificados, buscando la mejoría de la calidad de la prescripción y el fortalecimiento de la asistencia en el SUS.

Palabras clave: Prescripciones. Uso de medicamentos. Salud pública.

\section{INTRODUÇÃO}

Os medicamentos são considerados a tecnologia terapêutica mais utilizada no controle das doenças e produzem o aumento da expectativa e da qualidade de vida da população, sendo que a ausência deles, ou uso irracional, pode ocasionar risco de dano desnecessário associado ao cuidado de saúde. Além disso, eles afetam negativamente os investimentos nas ações de saúde, visto que os gastos públicos com medicamentos são crescentes e constituem o segundo maior item de despesa dos sistemas de atenção à saúde, somente superados pela atenção hospitalar. ${ }^{(1,2)}$

No Sistema Único de Saúde (SUS), o acesso da população aos medicamentos se dá através da prescrição, sendo um processo de escolha do prescritor a indicação de uma terapêutica medicamentosa adequada para o usuário e condutas a serem adotadas, após diagnóstico preciso e fundamentado na avaliação do estado geral de saúde do paciente. . $^{(3,4,5)}$

Dessa forma, a prescrição exerce papel ímpar na prevenção de erros de medicação, uma vez que é a partir dela que todo o fluxo medicamentoso de tratamento vai chegar ao usuário. Pesquisas apontam que erros de medicação geram custos diretos e indiretos ao SUS e à economia do país, estimando-se que a prescrição incorreta possa acarretar gastos de 50 a $70 \%$ a mais nos recursos governamentais destinados aos medicamentos. Esses dados são alarmantes, tendo em vista que a utilização 
de medicamentos no Brasil tem sido considerada exacerbada, indiscriminada e, muitas vezes desnecessária, impostas pela valorização da medicalização empregada por usuários e profissionais há vários anos. Isso demonstra que, além de despesas orçamentárias, é colocada em risco a vida das pessoas. ${ }^{(6,7)}$

Portanto, as prescrições devem seguir alguns critérios para garantir a correta compreensão das informações, conforme a Lei Federal n 5.991 de 1973 e a Lei Municipal para João Pessoa n 1.414 de 2014, incluindo: legibilidade, identificação do usuário, nomenclatura oficial do fármaco prescrito por Denominação Comum Brasileira (DCB) ou quando for ausente, utilizar a Denominação Comum Internacional (DCI), forma farmacêutica, posologia, via de administração, duração do tratamento e data de emissão da receita. Também deve apresentar os dados do prescritor como nome, assinatura, carimbo e número de inscrição no Conselho Regional da Categoria. ${ }^{(8,9)}$

Desse modo, tornam-se importantes estudos regionais que priorizem e enfatizem a análise de erros mais frequentes e rotineiros em prescrições, objetivando a diminuição dos mesmos e promovendo maior segurança ao usuário, buscando o uso racional do medicamento, sensibilização dos prescritores e evitando gastos desnecessários à saúde pública.

Portanto, este trabalho buscou avaliar prescrições dispensadas pela farmácia da Unidade de Saúde da Família (USF) Nova Conquista, localizada no município de João Pessoa, na Paraíba (PB), segundo critérios estabelecidos pelas Normativas $n^{\circ} 5.991$ de 1.973 e a Lei Municipal de João Pessoa nº 1.414 de $2014 .{ }^{(8,9)}$

\section{MATERIAL E MÉTODOS}

\section{Tipo de estudo e amostra}

A pesquisa proposta se caracterizou como um estudo transversal retrospectivo descritivo, com abordagem quantitativa, com a finalidade de observar, descrever e documentar aspectos da situação estudada. Apresenta-se como uma fotografia ou corte instantâneo que se faz em uma população, por meio de uma amostragem a partir de registros do passado. ${ }^{(10,11)}$

A amostragem foi constituída pelas prescrições legíveis e aviadas por médicos, dentistas e enfermeiros e dispensadas pela farmácia da USF Nova Conquista localizada na cidade de em João Pessoa (PB), que é composta de quatro Equipes Saúde da Família (Mateus II, Mateus III, Mateus IV e Mateus V), atendendo a aproximadamente 10 mil usuários do território do bairro Alto do Mateus. A farmácia da USF Nova Conquista recebe mensalmente uma média de 1.213 prescrições, para obtenção de uma amostra representativa, foi utilizado cálculo segundo Barbetta $(2006)^{(12)}$, em que é possível contabilizar uma primeira aproximação do tamanho da amostra, utilizando o erro amostral, sendo o erro amostral utilizado nesta pesquisa de 5\%. Diante disso, a amostragem do presente estudo resultou em aproximadamente 300 prescrições analisadas (com 721 medicamentos prescritos). O período de análise foi o mês de setembro de 2015, utilizando prescrições arquivadas na farmácia da USF Nova Conquista.

\section{CRITÉRIOS DE INCLUSÃO E EXCLUSÃO}

A pesquisa incluiu prescrições legíveis de medicamentos aviadas por médicos, dentistas e enfermeiros. Foram excluídas todas as prescrições ilegíveis ou fora do período de coleta de dados selecionado.

\section{MÉTODO PARA AVALIAÇÃO DAS PRESCRIÇÕES}

De acordo com regras sobre a prescrição de medicamentos, mediante aspectos éticos a serem seguidos pelos profissionais envolvidos no ato da prescrição, se apresentam as Normativas nº 5.991 de 1973 e a Lei Municipal de João Pessoa n 1.414 de 
2014, que dispõem sobre critérios que devem estar presentes na prescrição de medicamentos, de forma a promover o seu uso racional, conforme mostra a Figura $1 .^{(8,9)}$

Figura 1. Representação dos critérios para avaliação das prescrições segundo Legislações Federal e Municipal

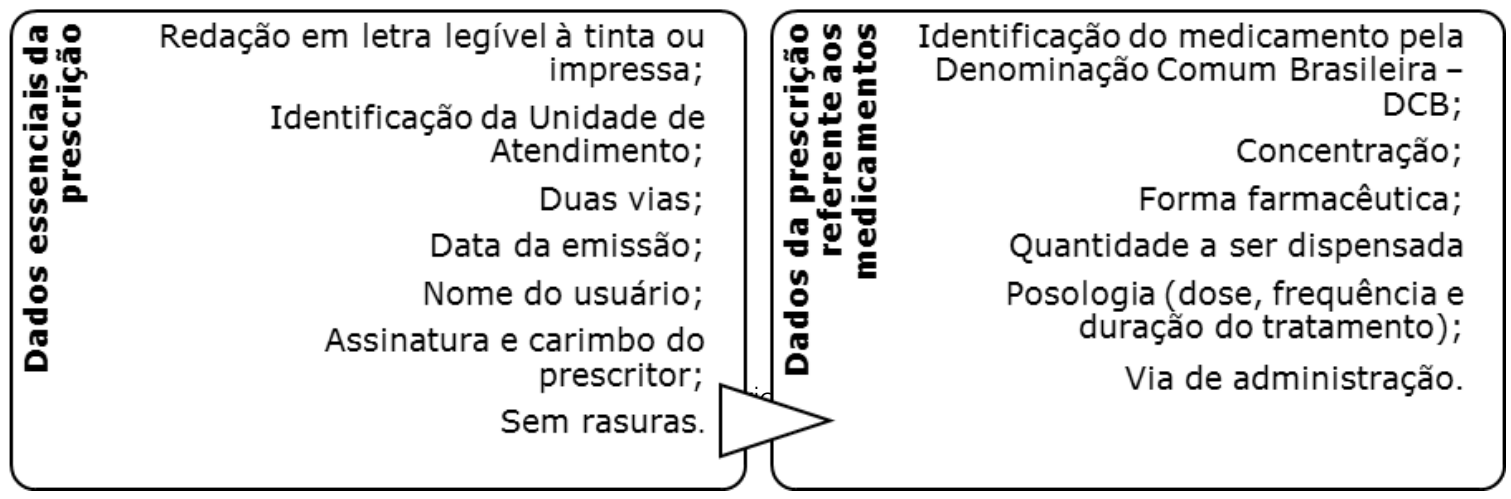

Fonte: Adaptado de Brasil (1973) e João pessoa $(2014)^{(8,9)}$

Os dados quantitativos da prescrição foram submetidos a cálculos de porcentagem em planilhas Excel. O presente estudo foi submetido ao Comitê de Ética e desenvolvido após sua aprovação, segundo protocolo 51642215.3.0000.5573, garantido o anonimato dos prescritores e usuários conforme Resolução do Conselho Nacional de Saúde n 196 de 1996 e suas atualizações.

\section{RESULTADOS E DISCUSSÃO}

Dos 721 medicamentos prescritos, observaram-se prescrições contendo de 1 a 5 medicamentos (Tabela 1), com média de 2,4 medicamentos por prescrição. Valores semelhantes foram encontrados em estudos realizados por Bandeira e colaboradores $(2015)^{(13)}$ e Souza e colaboradores (2012)(14), com média de medicamentos/prescrição de 2,43 e 2,4 respectivamente.

Tabela 1. Número de medicamentos nas prescrições coletadas

\begin{tabular}{ccc}
\hline $\mathbf{N}^{\circ}$ de medicamentos/prescrição & Número de prescrições $\mathbf{( n = 3 0 0 )}$ & \% (100) \\
\hline 01 & 1 & $0,30 \%$ \\
02 & 200 & $66,70 \%$ \\
03 & 79 & $26,33 \%$ \\
04 & 17 & $5,67 \%$ \\
05 & 3 & $1 \%$ \\
\hline
\end{tabular}

Fonte: Dados da pesquisa (2016).

Os valores constatados neste estudo apresentaram-se sem tendência para polimedicação, baseado nos valores propostos pela Organização Mundial de Saúde, que estabelece como polimedicação três ou mais medicamentos por prescrição. A polimedicação ou polifarmácia pode contribuir para o uso de medicamentos inadequados e administração errônea, devido a 
tratamentos com esquemas terapêuticos mais complexos. Isso cria uma barreira à adesão ao tratamento e, além disso, favorece a chance de desenvolvimento de reações adversas, em função do potencial risco de interações medicamentosas.(15, 16, 17)

Quanto aos profissionais prescritores, a predominância foi para enfermeiros com $52 \%$, seguido por médicos $44 \%$ e, em menor porcentagem, dentistas com 3\%. Prescrições que não obtiveram identificação, devido à falta do carimbo e assinatura do prescritor, corresponderam a $1 \%$.

O maior percentual dos resultados deste estudo para prescrição realizada por enfermeiros ocorre embasado na Lei do exercício profissional desta classe (Lei $n^{\circ} 7.498$ de 1986), a qual regulamenta a prescrição de medicamentos praticada pelo enfermeiro como integrante da equipe saúde da família. Esse profissional tem autonomia na escolha dos medicamentos e respectiva posologia, respondendo integralmente pelos atos praticados e respeitando os limites legais para prática desta ação (Programas de Saúde Pública e rotinas previamente aprovadas em instituições de saúde, pública ou privada).(18)

Todas as prescrições analisadas continham medicamentos escritos à mão. Essa variável está diretamente relacionada com a legibilidade das prescrições, podendo refletir em erros de dispensação. De acordo com Bates (2000)(19) e Silva, Passos e Carvalho (2012)(20), a mudança da prescrição feita manualmente pela prescrição digitalizada, ou totalmente eletrônica, representa uma importante estratégia para diminuição erros encontrados na prescrição, evitando eventuais dúvidas, rasuras e, ampliando a segurança ofertada ao usuário. Contudo, observou-se que ainda há dificuldades para implantação desse sistema eletrônico nas USF's, principalmente devido ao alto custo de programas específicos às instituições públicas. Portanto, é imprescindível a cooperação dos prescritores para que se consiga atingir a padronização das receitas de forma legível e que sejam abertos ao diálogo, para dirimir quaisquer dúvidas referentes a elas.

Com relação às informações essenciais que devem estar contidas na prescrição, observa-se na Tabela 2 que as principais falhas dizem respeito à: ausência do endereço do usuário na prescrição (98\% dos casos) e presença de abreviaturas (96,77\%).

Tabela 2. Descrição das características das prescrições

\begin{tabular}{|c|c|c|c|}
\hline \multicolumn{2}{|c|}{ Variável } & \multirow{2}{*}{$\begin{array}{c}\text { Prescrições } \\
(\mathbf{n}=\mathbf{3 0 0})\end{array}$} & \multirow{2}{*}{$\begin{array}{c}\text { Valor em \% } \\
74 \%\end{array}$} \\
\hline & Presente & & \\
\hline Identıtıcaçao Unidade de saude & Ausente & 78 & $26 \%$ \\
\hline \multirow{2}{*}{ Duas vias } & Presente & 243 & $81 \%$ \\
\hline & Ausente & 57 & $19 \%$ \\
\hline \multirow{2}{*}{ Data } & Presente & 300 & $100 \%$ \\
\hline & Ausente & 0 & $0 \%$ \\
\hline \multirow{2}{*}{ Nome usuário } & Presente & 299 & $99,67 \%$ \\
\hline & Ausente & 1 & $0,33 \%$ \\
\hline \multirow{2}{*}{ Endereço Usuário } & Presente & 6 & $2 \%$ \\
\hline & Ausente & 294 & $98 \%$ \\
\hline \multirow{2}{*}{ Assinatura do prescritor } & Presente & 298 & $99,33 \%$ \\
\hline & Ausente & 2 & $0,67 \%$ \\
\hline \multirow{2}{*}{ Carimbo } & Presente & 293 & $97,67 \%$ \\
\hline & Ausente & 7 & $2,33 \%$ \\
\hline \multirow{2}{*}{ Rasuras } & Presente & 21 & $7 \%$ \\
\hline & Ausente & 279 & $93 \%$ \\
\hline \multirow{2}{*}{ Abreviaturas } & Presente & 508 & $96,77 \%$ \\
\hline & Ausente & 17 & $3,23 \%$ \\
\hline
\end{tabular}

Fonte: Dados da pesquisa (2016). 
A ausência da segunda via (via carbonada), detectada em 19\% das prescrições, evidenciou a preocupação mediante a posologia prescrita, uma vez que o usuário retorna à sua residência apenas com o medicamento e informações verbais ditas pelo médico no momento da consulta e que facilmente podem ser esquecidas. Diante disto, torna-se essencial que o usuário esteja com uma via da prescrição, através da qual possa seguir a posologia e período de tratamento adequadamente.

O endereço do usuário (dado de presença obrigatória, conforme legislação vigente) esteve presente em apenas $2 \%$ das prescrições. Resultado muito próximo foi encontrado no estudo de Braga e Crespo (2015)(21), com ausência de endereço em $98,8 \%$. Segundo Aldrigue e colaboradores (2006)(22), este dado pode estar relacionado com o tempo reduzido da consulta médica e o baixo índice de informatização e, embora não implique em repercussões clínicas diretas, a ausência desse item pode comprometer a possibilidade de contato entre farmácia e paciente em situações pós-dispensação, em que isso se faça necessário.

Quanto à análise das abreviaturas, foram identificadas 525 abreviaturas na amostra de 300 prescrições, totalizando $96,77 \%$ das receitas. Contabilizou-se apenas um tipo de cada abreviatura nas prescrições, descartando repetições, sendo localizados 31 tipos de variações, algumas descrevendo o mesmo item, como por exemplo, para denominar comprimido: CP, COMP, COMPR, COP, CPS, COMPS e COPS. Este resultado corrobora com outros estudos ${ }^{(23,24,25,26)}$ que apresentam percentual de abreviaturas superior a $90 \%$.

Para Araújo et al. (2014)(27), a frequente utilização de abreviaturas faz parte da prática cotidiana de prescritores, sendo justificada pela economia de tempo. Entretanto, pode ter efeito oposto quando gera dificuldade de interpretação à equipe assistencial. Em outro estudo realizado por Rosa e colaboradores (2009)(28), evidencia-se que a falta de padronização e o frequente uso de abreviaturas demonstram falhas latentes que podem contribuir para ocorrência de erros de medicação, por seu potencial de confusão e falhas de comunicação, sendo antiga a ideia de eliminar o seu uso.

Com relação às informações de presença obrigatória na prescrição do medicamento (Tabela 3), observaram-se falhas em todas as variáveis analisadas, sendo a ausência de descrição da forma farmacêutica $(91,12 \%)$ e da duração do tratamento (76,98\%), os principais erros de prescrição detectados.

Tabela 3. Descrição das características relacionadas aos medicamentos

\begin{tabular}{|c|c|c|c|}
\hline Variável & & $\begin{array}{c}\text { Medicamentos } \\
\text { prescritos }(n=721)\end{array}$ & $\%(100)$ \\
\hline \multirow{3}{*}{ Concentração } & Presente & 214 & $68,52 \%$ \\
\hline & Ausente & 494 & $29,68 \%$ \\
\hline & Inadequada & 13 & $1,80 \%$ \\
\hline \multirow{2}{*}{ Forma farmacêutica } & Presente & 64 & $8,88 \%$ \\
\hline & Ausente & 657 & $91,12 \%$ \\
\hline \multirow{10}{*}{ Via de administração } & Oral & 636 & $88,21 \%$ \\
\hline & Tópico & 27 & $3,74 \%$ \\
\hline & Interno & 16 & $2,22 \%$ \\
\hline & Externo & 8 & $1,10 \%$ \\
\hline & Vaginal & 5 & $0,70 \%$ \\
\hline & Ginecológico & 3 & $0,42 \%$ \\
\hline & Inalatória & 7 & $0,98 \%$ \\
\hline & Injetável & 2 & $0,28 \%$ \\
\hline & Subcutânea & 4 & $0,55 \%$ \\
\hline & Ausente & 13 & $1,80 \%$ \\
\hline
\end{tabular}


Continuação da Tabela 3.

\begin{tabular}{cccc}
\hline \multirow{2}{*}{ Dose } & Presente & 684 & $94,87 \%$ \\
& Ausente & 37 & $13 \%$ \\
& Prequente & 587 & $81,41 \%$ \\
& Ausente & 134 & $18,59 \%$ \\
& Presente & 166 & $23,02 \%$ \\
Duração & Ausente & 555 & $76,98 \%$ \\
\hline \multirow{2}{*}{ Quantidade a ser dispensada } & Presente & 695 & $96,40 \%$ \\
& Ausente & 26 & $3,6 \%$
\end{tabular}

Fonte: Dados da pesquisa (2016).

Estudo realizado por Mastroianni (2009)(29) aponta que a falta de informações essenciais sobre o medicamento, tais como: concentração, forma farmacêutica, via de administração e posologia (dose, frequência e duração do tratamento), pode levar ao desperdício de recursos financeiros, além de prejuízo terapêutico ao usuário. Isso implica em tratamentos inadequados e ineficazes, podendo refletir uma possível diminuição da qualidade do atendimento realizado pelo prescritor, em uma dispensação errônea ou em um tratamento de difícil adesão para o usuário, devido à ausência de informações necessárias para sucesso na terapêutica medicamentosa.

Ferreira e Ferreira $(2015)^{(30)}$ refletem ideia semelhante, na qual se observa que, quando essas informações estão ausentes, ilegíveis ou incompletas, aumenta a ocorrência de erros na dispensação e utilização dos medicamentos, podendo inclusive, elevar as taxas de mortalidade em decorrência de tratamentos ineficazes e pouco seguros. Pode também direcionar o paciente à atenção secundária e terciária de saúde, acarretando maiores gastos à saúde pública. Uma vez que os medicamentos disponibilizados pelo SUS não são acompanhados de bulas, tais informações tornam-se de suma importância para o paciente, por isso devem estar de forma clara e legível na prescrição, evitando as informações inadequadas ou que fiquem subentendidas ao usuário.

Com relação à nomenclatura, observou-se adequação à DCB em 84,47\% das prescrições, conforme determina a legislação. Porém, 10,40\% dos medicamentos encontravam-se escritos como abreviações do seu nome, 5,55\% estavam descritos como medicamentos de referência e 1,94\% na nomenclatura de medicamento similar. Resultados superiores foram verificados em estudos de Valadão e colaboradores $(2009)^{(31)}$ e Freitas e Nobre (2011) ${ }^{(32)}$ com resultados de $86 \%$ e $91 \%$ respectivamente e valores inferiores foram encontrados em estudo de Santos e Nitriti (2004)(33), revelando resultado de $30,6 \%$ de medicamentos prescritos na DCB. Tais divergências encontradas podem refletir na utilização de critérios diferentes entre os pesquisadores, assim como um perfil diferenciado de padrão de comportamento dos prescritores nas diferentes regiões estudadas. É importante salientar que, no âmbito do SUS, todas as prescrições devem ser realizadas pelos nomes das substâncias ativas ou genéricas na forma de DCB e, na sua ausência, na DCl, já que o uso da nomenclatura de medicamento de marca ou similar pode levar a erros de prescrição e dispensação, pois os nomes comerciais variam dentre as indústrias produtoras e estão sujeitos a mudanças, atendendo a interesses de marketing do mercado. . $^{(34)}$

$\mathrm{Na}$ análise dos medicamentos mais prescritos (Tabela 4), os resultados evidenciaram que anti-inflamatórios, como por exemplo, a dipirona e o ibuprofeno, são a classe mais prescrita, seguido por anti-hipertensivos, antialérgicos e antimicrobianos. 
Tabela 4. Descrição da classe farmacológica dos medicamentos prescritos

\begin{tabular}{lcc}
\hline CLASSES DE MEDICAMENTOS & $\mathbf{n = 7 2 1}$ & $\mathbf{\%} \mathbf{( 1 0 0 )}$ \\
\hline ANTI-INFLAMATÓRIO NÃO ESTEROIDE & 202 & $28,04 \%$ \\
\hline ANTI-HIPERTENSIVO & 102 & $14,15 \%$ \\
\hline ANTIALÉRGICO & 83 & $11,51 \%$ \\
\hline ANTIBIÓTICO & 63 & $8,73 \%$ \\
\hline COMPLEMENTO VITAMÍNICO & 39 & $5,40 \%$ \\
\hline ANTI-INFLAMATÓRIO ESTEROIDAL & 32 & $4,44 \%$ \\
\hline EXPECTORANTE/MUCOLÍTICO & 31 & $4,30 \%$ \\
\hline HIPOGLICEMIANTE ORAL & 29 & $4,02 \%$ \\
\hline ANTIAGREGANTE PLAQUETÁRIO & 28 & $3,88 \%$ \\
\hline ANTIFÚNGICO VAGINAL & 14 & $1,94 \%$ \\
\hline ANTI-HELMÍNTICO/ANTIPROTOZOÁRIO & 14 & $1,94 \%$ \\
\hline ANTIESPASMODICO & 13 & $1,80 \%$ \\
\hline ANTIEMÉTICO & 12 & $1,66 \%$ \\
\hline ANTIFÚNGICO & 11 & $1,53 \%$ \\
\hline ANTIARRITMICO/CARDIOTÔNICO & 10 & $1,38 \%$ \\
\hline ANTIFLATULENTO & 9 & $1,25 \%$ \\
\hline HIPOGLICEMIANTE (INSULINA) & 7 & $0,97 \%$ \\
\hline ANTICONCEPCIONAL & 6 & $0,83 \%$ \\
\hline BRONCODILATADOR & $6,83 \%$ \\
\hline ANTIULCEROSO & 6 & $0,70 \%$ \\
\hline REPOSITORES HIDROELETROLÍTICOS & 5 & $0,42 \%$ \\
\hline ESCABICIDA & 3 & $0,28 \%$ \\
\hline & 2 &
\end{tabular}

Fonte: Dados da pesquisa (2016)

Além do alto percentual de prescrições contendo anti-inflamatórios não-esteroides (AINES), em 2,91\% das prescrições estavam presentes dois medicamentos dessa mesma classe farmacológica, tornando essas prescrições potencialmente perigosas, pois o uso de forma incorreta, com superdose ou por tempo indevidamente prolongado desses medicamentos, pode representar riscos à saúde dos usuários. É possível haver interações medicamentosas que causem efeitos adversos como: irritação na mucosa gastrointestinal inferior, lesão hepática, indução de neutropenia, efeitos na pressão arterial, efeitos pulmonares, inibição plaquetária, reações de hipersensibilidade e efeitos no sistema nervoso central. ${ }^{(35)}$

Segundo Guzatto e Bueno (2007)(34), uma possível estratégia para sensibilização dos prescritores sobre os erros de prescrição seria o desenvolvimento de trabalhos em educação permanente dentro da USF, que tenha como objetivo reverter erros e omissões. Isso evitaria: trocas de medicamentos, duplicidade terapêutica e substituição de formas farmacêuticas no momento da dispensação, administração de medicamentos na via errada, dose errada, uso de medicamento com duração de tratamento equivocada e fraudes/falsificação das receitas. 


\section{CONCLUSÃO}

O presente trabalho revela que as prescrições avaliadas não atenderam à totalidade dos critérios de avaliação propostos neste estudo, indicando que alguns hábitos de prescrição devem ser modificados em busca da melhoria da qualidade da prescrição. Fazendo-se necessário uma maior conscientização dos prescritores, de forma a tornar a prescrição a mais correta possível, atendendo o que é determinado na legislação e, possibilitando ao usuário atendimento pleno e eficaz, pois erros de prescrição e interações medicamentosas se tornam dispendiosos para o SUS.

Dessa forma, são necessárias estratégias que busquem melhorar a promoção de saúde na atenção básica, como trabalhos de educação permanente e que visem sensibilizar os prescritores e demais atores envolvidos neste processo sobre o ato de prescrever medicamentos. Também é necessária a presença do farmacêutico na USF, possibilitando uma adequada dispensação dos medicamentos e a devida interpretação da prescrição, para maior benefício ao usuário que procura a atenção básica, além de proporcionar o fortalecimento da assistência farmacêutica no SUS.

\section{REFERÊNCIAS}

1. Mendes, E. V. As redes de atenção à saúde. 2. ed. Brasília: Organização Pan-Americana da Saúde, 2011, p. 549.

2. Correr, C. J. Brasil. Ministério da Saúde. Serviços Farmacêuticos na Atenção Básica à saúde. Cuidado do Farmacêutico na Atenção Básica. O medicamento enquanto insumo essencial das ações de saúde. Brasília - DF. 2014.

3. Portaria N. 3.916 de 30 de outubro de 1998. Aprova a Política Nacional de Medicamentos. Diário Oficial da União (Brasília). 1998 Out 30.

4. Rosa, M.B. et. al. Erros na prescrição hospitalar de medicamentos potencialmente perigosos. Revista de Saúde Pública. 2009; v. 43, n. 3, p. 490-498.

5. Bandeira, V. A. C., et al. Análise das prescrições dispensadas em uma unidade básica de saúde do município de ljuí - RS. Saúde (Santa Maria). 2015; v. 41, n. 1, jan./jul., p. 229-238.

6. Ev, L. S.; Guimarães, A. G.; Castro V. S. Avaliação das prescrições dispensadas em uma Unidade Básica de Saúde do Município de Ouro Preto, Minas Gerais, Brasil. Latin. American Journal of Pharmacy. 2008; v. 28, n. 4, 2008.

7. Ferrari, C. K. U. et al. Falhas na Prescrição e Dispensação de Medicamentos Psicotrópicos: Um problema de Saúde Pública. Revista de Ciências Farmacêuticas Básica e Aplicada. 2013; v. 34, n. 1, p. 109-116.

8. Lei $n^{\circ}$. 5.991, de 17 de dezembro de 1973. Dispõe sobre o controle sanitário do comércio de drogas, medicamentos, insumos farmacêuticos e correlatos, e dá outras providências. Diário Oficial da União (Brasília). 1973 Dez. 17.

9. Lei $n^{\circ} 1414$, de 02 a 08 de março de 2014. Dispõe sobre critérios para promover o uso racional de medicamentos na rede municipal de saúde. 2014.

10. Fletcher, R. H; Fletcher, S. W; Wagner, E. H. Epidemiologia clínica: elementos essenciais. Porto Alegre: Artmed; 2003.

11. Pollit D. F.; Beck C. T.; Hungler B. P. Fundamentos de pesquisa em enfermagem: métodos, avaliação e utilização. 5. ed. Porto Alegre: Artmed; 2004.

12. Barbetta, P. A. Estatística aplicada às ciências sociais. 6. ed. Florianópolis: EdUFSC, 2006.

13. Bandeira, V. A. C., et al. Análise das prescrições dispensadas em uma unidade básica de saúde do município de ljuí - RS. Saúde (Santa Maria). 2015; v. 41, n. 1, jan./jul., p. 229-238. 
14. Souza, J. M. et al. Avaliação dos indicadores de prescrição e da demanda atendida de medicamentos no Sistema Único de Saúde de um município do Sul do Estado de Santa Catarina. Revista de Ciência Farmacêutica Básica Aplicada. 2012; v. 33, n. 1, p. 107-113.

15. OMS. Organización Mundial de la Salud. Como investigar el uso de medicamentos en los servicios de salud. Indicadores seleccionados del uso de medicamentos. Ginebra; 1993. 100p.

16. Acurcio, F. A. et al. Complexidade do regime terapêutico prescrito para idosos. Revista de Associação Medica Brasileira. $2009 ;$ v. 55, p. 468-74.

17. Sousa, R. P. M. et al. Avaliação do padrão da prescrição médica, segundo os indicadores da OMS para o uso racional de medicamentos nas unidades básicas de saúde de Santarém (PA). Revista EM FOCO - Fundação Esperança/IESPES. 2016; v. 1, n. 25, p. 4-18.

18. Lei n. 7.498, de 25 de junho de 1986: dispõe sobre a regulamentação do exercício da enfermagem e dá outras providências. Diário Oficial da União (Brasília). 1986. jun. 26.

19. Bates, D.W. Improving medication safety across institutions. J Qual Improvem. 2000; v.26, n. 6, p. 319-320.

20. Silva, L. D. Passos, R.S, Carvalho, M. F. CARACTERÍSTICAS E EVIDÊNCIAS DA PRODUÇÃO CIENTíFICA DE ENFERMEIROS SOBRE ERROS DE MEDICAÇÃO NO AMBIENTE HOSPITALAR. Revista Rene. 2012; v. 12, n. 2, p. 480-91.

21. Braga, H. S. Crespo, J. M. R. S. Estudo farmacoepidemiológico sobre medicamentos antimicrobianos na cidade de Muriaé (MG): uma análise dos aspectos legais das prescrições Revista Científica da Faminas. 2015; v. 11, n. 2.

22. Aldrigue, R. F. T. et al. Análise da completude de prescrições médicas dispensadas em uma farmácia comunitária de Fazenda Rio Grande - Paraná (Brasil). Acta. Farm. Bonaerense. 2006; v. 25, n. 3.

23. Oliveira, R. C. et al. Estratégias para prevenção de erros de medicação no setor de emergência. Rev. Bras. de Enferm. 2005; v. 58, n. 4 , p. 399-404.

24. Gimenes, F. R. et al. Medication wrong-route administrations in relation to medical prescriptions. Rev. Latino-Am. Enfermagem. 2011; v. 19, n. 1, p. 11-17.

25. Araujo, A. E. P. et al. ANÁLISE DE PRESCRIÇÕES EM MATERNIDADE PÚBLICA BRASILEIRA. Revista Brasileira de Farmácia Hospitalar e Serviços de Saúde. 2014; v. 5 n. 2, p. 63-68.

26. Lopes, L. N. et al. Qualidade das prescrições médicas em um Centro de Saúde Escola da Amazônia Brasileira. Revista da Sociedade Brasileira Clínica Médica. 2014; v. 12, n. 2, abr./jun.

27. Araujo, A. E. P. et al. ANÁLISE DE PRESCRIÇÕES EM MATERNIDADE PÚBLICA BRASILEIRA. Revista Brasileira de Farmácia Hospitalar e Serviços de Saúde. 2014; v. 5 n. 2, p. 63-68.

28. Rosa, M.B. et al. Erros na prescrição hospitalar de medicamentos potencialmente perigosos. Revista de Saúde Pública. 2009; v. 43, n. 3, p. $490-498$.

29. Mastroianni, P. Análise dos aspectos legais das prescrições de medicamentos. Revista de Ciências Farmacêuticas Básica e Aplicada. 2009; v. 30, n. 2, p. 45-48.

30. Ferreira, T. A. Ferreira, C. F. D. QUALIDADE DA PRESCRIÇÃO DE ANTIMICROBIANOS COMERCIALIZADOS NA REGIÃO NOROESTE DO PARANÁ, BRASIL. SaBios. 2015; v. 10, n. 1, p. 131-137. 
31. Valadão, A. F. et al. Prescrição médica: um foco nos erros de prescrição. Revista Brasileira de Farmácia. 2009; v. 90, n. 4, p. 340-343.

32. Freitas, J. M. S. M. Nobre, A. C. L. AVALIAÇÃO DA ASSISTÊNCIA FARMACÊUTICA DO MUNICÍPIO DE MOMBAÇA-CE. Revista Brasileira de Farmácia Hospitalar e Serviços de Saúde. 2011; v. 2, n. 1, p. 15-20.

33. Santos, V. Nitrini, S. M. O. O. Indicadores do uso de medicamentos prescritos e de assistência ao paciente de serviços de saúde. São Paulo. Revista de Saúde Pública. 2004; v. 38, n. 6, p. 819-826.

34. Guzatto, P.; Bueno, D. Análise de prescrições medicamentosas dispensadas na farmácia de uma unidade básica de saúde de Porto Alegre - RS. Revista HCPA. 2007; v. 27, n. 3.

35. Mota, P. M. et al. Estudo sobre a utilização de antiinflamatórios não esteroidais prescritos em receitas para idosos da região Noroeste Paulista. Revista de Ciência Farmacêutica Básica e Aplicada. 2010; v. 31, n. 2, p. 157-163.

Submetido em: 05/12/2016 Aceito em: 30/08/2017 Publicado em: 31/12/2017 